Ann. Biol. anim. Bioch. Biophys., I963, 3 (3), 207-218

\title{
HYPOTRICHOSE PÉRIODIQUE CHEZ LA SOURIS
}

\author{
N. KOBOZIEFF, Nathalie POMRIASKINSKY-KOBOZIEFF et Eugénie GEMÄILING \\ Laboratoire de Génétique, École nationale vétérinaire, Alfort (Seine)
}

\section{SOMMAIRE}

Cette étude de l'hypotrichose périodique a porté sur 2985 souris $\mathrm{c}_{57} \mathrm{BL} / \mathrm{J} a x 6$.

L'anomalie se manifeste entre le $16^{\mathrm{e}}$ et le $24^{\mathrm{e}}$ jour après la naissance; elle se caractérise par une raréfaction ou une chute des poils sur tout ou partie du corps des sujets atteints et s'accompagne d'un arrêt de croissance - on note une différence pondérale qui peut atteindre $5 \mathrm{~g}$ entre les sujets atteints et les sujets normaux d'une même portée. L'épilation débute le plus souvent dans la région scapulaire et progresse par vagues sucessives vers la base de la queue. Le phénotype est variable, on distingue 6 formes de première mue anormale :

- épilation faible, poils clairsemés sur tout ou partie du corps (Type I),

- épilation de la région scapulaire (Type II),

- épilation s'étendant sur toute la région thoracique (Type III),

- épilation s'étendant jusqu'à la région lombaire incluse (Type IV), queue (Type $\mathrm{V}$ ),

- épilation exclusivement cantonnée au niveau des cuisses (Type VI).

On observe parfois une deuxième, troisième et même quatrième épilation à l'occasion des autres mues, mais elles sont nettement plus rares et se manifestent de façon très irrégulière.

Les 9 types de croisements effectués ont permis d'établir qu'il s'agit d'une mutation récessive à récessivité incomplète et à faible pénétrance.

L'analyse génétique de la descendance normale, issue de deux anormaux (pénétrance 30, I p. 100) montre que ces sujets " normal overlaps" se comportent comme des anormaux ; dans le croisement $\mathrm{A} n \times \mathrm{N} o v$ la pénétrance est légèrement plus faible (24 p. 100), elle n'est que de $2 \mathrm{I}, 5 \mathrm{p}$. Ioo dans le croisement $\mathrm{N} o v \times \mathrm{N} o v$, issus de deux anormaux et en $\mathrm{F}_{2} \mathrm{~N} o v \times \mathrm{F}_{2} \mathrm{~N} o v$ de $9,7 \mathrm{p}$. 100 seulement, en raison de l'intervention de gènes modificateurs inhibiteurs.

Par ailleurs. les facteurs "parturition" et "saison" ont une influence certaine sur la fréquence d'apparition de l'anomalie.

\section{INTRODUCTION}

L'hypotrichose ou atrichose (alopécie partielle ou totale), juvénile ou périodique, associée ou non à d'autres affections, se rencontre assez fréquemment dans différentes espèces — elle est d'origine spécifique ou mutationnelle, héréditaire ou non (accidentelle ou provoquée). 
C'est chez la Souris (Mus musculus, Mus molossinus, Peromyscus maniculatus gambeli), domestique ou sauvage, que les anomalies d'ordre mutationnel se rencontrent le plus souvent. On dénombre à ce jour dans la littérature une dizaine de mutations qui sont soit dominantes, soit récessives.

Parmi les mutations dominantes on relève : Naked $(\mathrm{N})$, signalée par LEBEDINSKY et Dauwart (I927) et EbBenhorst TeNGbergen (I939), Alopécia (A1), étudiée par DiCkie, KELTON, FIELdER, INGalias et SNELI, (I949) et Dickie (I955), enfin par Hunsicker (Ig60).

Parmi les mutations récessives GoRdon (r850), fut, semble-t-il, le premier à signaler une alopécie chez la souris Mus musculus, Sumner (1924), a observé une mutation " hairless" chez les souris. Peromyscus maniculatus gambeli, étudiée histologiquement par Rigdon et Packchanian (1957); chez la souris Mus musculus, la mutation " hairless " (hr), découverte par BROOKE (I926), fut étudiée ultérieurement par CREw (I927), CREw et MrRskaia (I932), David (I93I et I932) ; hypotrichosis juvenilis $(h i)$, décrite pas LOEFFLER (I934), rhino $\left(h r^{r h}\right)$, décrite par HowARD (I940) et Fraser (I946), de même que Steinberg et Fraser (I946), ont étudié la mutation hairless ( $h r)$, Ichtiosis (ic), mutation décrite par CARTER et PHILIPS (I949). Bald $\left(h r^{b a}\right)$, décrite par GARBER (I952); furless; $(f s)$, signalée par GREEN (I954); tufted — hereditary hairloss $(t f)$, observée par LYON (I956); ragged, mutation décrite par SLEE (I957) ; alopécia, décrite par KoBOzIEFF PomRIASKINSKY-KobOzIEFF et DRIEUX (1958) - alopécie constante, phénotypiquement semblable à celle décrite par HoWARD $\left(h \gamma^{r h}\right)$ - les tests pour l'allélomorphisme n'ont pas encore été pratiqués, et enfin calvino, citée d'après Mouse News Letter $n^{\circ}$ I7 (I957) comme recherche en cours de Pizarro.

Dans les autres espèces, nous relevons chez le Rat une hypotrichose héréditaire décrite par RoBERTS (I 924$)$, naked $(n)$, hairless $(h r)$, et cowlik (cw), mutations décrites par Castlf, Dempster et Shurrager (i955). Chez la Taupe, Furlotti (igio), décrit un cas isolé de malformation du pelage. Chez les Bovins, MoHR et WRIED'T (I928), décrivent une mutation récessive et létale, hypotrichosis congenita, dans la race Holstein-Friesan ; CoLE (I9I9), a observé un cas d'anomalie semblable dans la même race et WIPRECHT et HorLACHER (I935), dans la race Jersey. C'est aussi dans la race Holstein-Friesan que EISELE (I936), signale une alopécie récessive et létale, cette même anomalie est observée par REGAN, MEAD et GREGORY (I935), dans un troupeau de la race Jersey ayant une étroite parenté avec celle de WIPPRECHT. CRAFT et BLIZZARD (I934), décrivent une mutation récessive simple, semihairlessness, ches les Bovins ; LIUTIKOV (I938), signale une mutation hairlessness ; SHibata et Ishinara (I949), signalent une alopécie chez les bovins japonais ; HuTT et SAUNDERs (I953), décrivent une mutation récessive, hairless, dans la race Guernesey ELDINGE et ATKESON (I953), décrivent une mutation, streaked hairlessness, dans la race Holstein-Friesan ; Drieux, Priovzeau, Thierry et M. L. Priouzeau (i950), signalent un cas d'hypotrichose congénitale chez le veau, enfin KRALI,INGER (I955), décrit une alopécie chez les bovins. BONNET (I892), signale une hypotrichose congénitale chez la Chèvre. Chez le Porc, la mutation hairlessness, a été signalée par Hart et SteEnbock (I9I8), puis par Roberts et Carrol L (I93I) qui la décrivent comme une mutation dominante; DAVID (I932), signale tne mutation hairless chez le porc mexicain. TEGETMEIER ( 1876 ), décrit le cas isolé d'un Cheval atteint d'atrichose. KISLOVSKY (I928), décrit une mutation naked, chez le Lapin. LETARD (I938), 
décrit une atrichose récessive chez le Chat et MELLEN (I939), décrit une alopécie chez le chat mexicain. Chez le Chien, LETARD (I930), signale une atrichose dominante et létale; SCHEUER et KOHN (IgII), signalent des cas d'hypotrichose chez le Chien et chez 1'Homme. Enfin de nombreux cas d'alopécie ou d'atrichose chez 1'Homme sont décrits par WAEISCH (IgIo), Fischer (IgIo), David (I93I), GILlepsie (I937) Brain (I938), Thorner (1940) et Gates (I948). Quelques cas d'alopécie chez différentes espèces furent rassemblés dans une étude générale par KoBozIEFF et POMRIASKINSKY-KOBOZIEFF (I 943 ).

Le présent article porte sur l'évolution de 1'hypotrichose périodique, ses diversités phénotypiques ainsi que sur son étude génétique.

\section{MATÉRIEL E'T MÉTHODE}

Nous avons disposé de deux portées de 5 petits chacune où tous les souriceaux étaient atteints d'hypotrichose. Ces animaux provenaient de parents de la lignée $\mathrm{C}_{57} \mathrm{BL}$ Jax 6 ; ils avaient été observés par le $\mathrm{D}^{\mathrm{r}}$ Paraf, en septembre 1960 , dans son élevage du Laboratoire Central de l'École Nationale Vétérinaire d'Alfort. Nous n'avons pas pu savoir si leurs parents avaient présenté la même anomalie. Ils paraissaient âgés de 3 semaines environ et présentaient une épilation de tout le corps, seules la tête et la base de la queue restaient couvertes de poils $\left(^{1}\right.$ ). Ces souriceaux ( I $\sigma^{*}$ et 4 qq pour l'une des portées et $4 \hat{\delta} \hat{\delta}$ et $I$ \& pour l'autre), ont repris un aspect normal, c'est-à-dire se sont couverts de poils, 5 jours plus tard. C'est à partir de ce matériel que nous avons entrepris l'étude de l'hypotrichose.

Parmi les animaux qui nous ont été remis, 5 ot et $\mathrm{I}$ \% atteints d'hypotrichose ont survécu jusqu'à l'âge de la reproduction ; 4 ôt $^{\star}$ ont servi ì des croisement avec des souris de lignées normales $\mathrm{MO} / \mathrm{K} o$ et $\mathrm{RIII} / \mathrm{K} o$ (n'ayant jamais présenté aucune anomalie du tégument) et I $\delta$ et I $q$ ont été croisés entre eux. Nous avons effectué 2 séries de croisements :

${ }_{\mathrm{I}}^{\mathrm{re}}$ série.

I) A $n$ (atteint d'hypotrichose) $\times \mathrm{N} \mathrm{RIII} / \mathrm{K} o$ ou N Mo/Ko (lignées normales),

II) $\mathrm{A} n \times \mathrm{F}_{1} \mathrm{~N}$ du croisement I,

III) $\mathrm{A} n \times \mathbf{F}_{b} \mathrm{~N}$ du croisement II,

IV) $\mathrm{F}_{b} \mathrm{~N}$ du croisement $\mathrm{I} \times \mathrm{F}_{b} \mathrm{~N}$ du croisement $\mathrm{I}$,

V) $\mathrm{F}_{b} \mathrm{~N}$ du croisement II $\times \mathrm{F}_{b} \mathrm{~N}$ du croisement II.

$2^{\mathrm{e}}$ série.

VI) A $n \times \mathrm{A} n$ (atteints d'hypotrichose),

VII) $A n \times \mathrm{F}_{1}$ Nov du croisement VI (normal overlaps),

VIII) $\mathrm{F}_{1} \mathrm{Nov}$ du croisement VI $\times \mathrm{F}_{1} \mathrm{~N} o v$ du croisement VI,

IX) $\mathrm{F}_{2} \mathrm{~N} o v$ du croisement VIII $\times \mathrm{F}_{2} \mathrm{~N} o v$ du croisement VIII.

\section{RÉSULTATS}

\section{Évolution des mues anormales}

D'une façon générale, les mues de souris normales ne se remarquent pas, les poils qui tombent étant remplacés au fur et à mesure par ceux qui repoussent. FRASER et NAY (I953), ont mis en évidence, à l'aide d'une méthode de teinture du poil,

(1) Nous tenons à remercier M. PARAF d'avoir bien voulu mettre à notre disposition ces spécimens précieux pour notre étude. 
les différents cycles des mues normales; plus particulièrement en ce qui concerne la $\mathrm{I}^{\mathrm{e}}$ mue (baby coat), ils ont remarqué que la mue progresse par vagues successives en partant de la base du crâne et en allant jusqu'à la base de la queue (FrASER, I95I).

Dans le cas de notre mutation, nous avons observé jusqu'à trois épilations successives à des périodes correspondant approximativement aux mues normales. A la naissance et jusqu'au $\mathrm{I} 6^{\mathrm{e}}-24^{\mathrm{e}}$ jour, les souris ne se distinguent pas des souris normales. A partir de ce moment l'épilation commence dans la région scapulaire omoplate droite ou gauche - laissant une zone dénudée, cet état reste stationnaire ou progresse, plus ou moins suivant le type, jusqu'à la base de la queue. Le degré d'épilation est variable. Pour plus de commodité nous avons classé les animaux en VI types différents d'après l'épilation de la face dorsale, l'épilation de la face ventrale étant moins nette et moins régulière.

Première épilation.

I. - Épilation très légère, pas de zone dénudée, poils clairsemés sur tout ou partie du corps.

II. - Épilation légère se contonnant à la région scapulaire et laissant une petite zone dénudée sur 1'omoplate gauche ou droite ou sur les deux.

III. - Épilation plus marquée, se propageant de la région scapulaire à toute la région thoracique.

IV. - Epilation plus nette gagnant progressivement sur les précédentes et atteignant la région lombaire à laquelle elle s'arrête - l'animal est dénudé au $3 / 4$.

V. Épilation presque complète, seules restent couvertes de poils la tête et la base de la queue.

VI. - Épilation exclusivement cantonnée au niveau des cuisses - le reste du corps est normalement couvert de poils.

Nous avons remarqué que l'épilation complète (Type V) est la plus rapide, la chute des poils s'opère en 2 à 4 jours. Il semble d'ailleurs que plus l'animal est sévèrement atteint et plus l'ensemble des phénomènes qui accompagnent l'épilation se produit rapidement. L'épilation la plus précice est celle de la région scapulaire elle apparaît entre le $\mathrm{I}_{4}$ et $\mathrm{I} \mathrm{G}^{\mathrm{e}}$ jour. L'épilation du 'Type VI, au contraire, apparaît tardivement — vers le $24^{\mathrm{e}}$ jour.

Les sujets atteints, une fois les poils tombés, demeurent couverts d'une peau rose, transparente, parfois légèrement ridée, pendant quelques jours, puis on observe un phénomène de pigmentation par vagues, avec des démarcations très nettes, c'est le début de la repousse des poils qui va progresser rapidement et d'autant plus rapidement que l'épilation aura été plus sévère. La repousse des poils s'opère dans le même ordre que celui observé pour leur chute - c'est à dire que les aires dénudées de la nuque (endroit où commence généralement l'épilation), sont les premières à se couvrir de poils courts, l'ordre de progression par vagues de la mue juvénile est ainsi respecté. Au cours de l'épilation, et surtout quand elle est très marquée, on observe un brusque arrêt de croissance, les animaux atteints sont nettement plus petits que les animaux normaux de la même portée (on observe des écarts de poids pouvant aller jusqu'à $5 \mathrm{~g}$ ). Au moment de la repousse des poils, on assiste à une reprise simultanée de la croissance. Au $35-40^{\mathrm{e}}$ jour les animaux atteints ne se distinguent plus des animaux normanx de la même portée. 
Première épilation
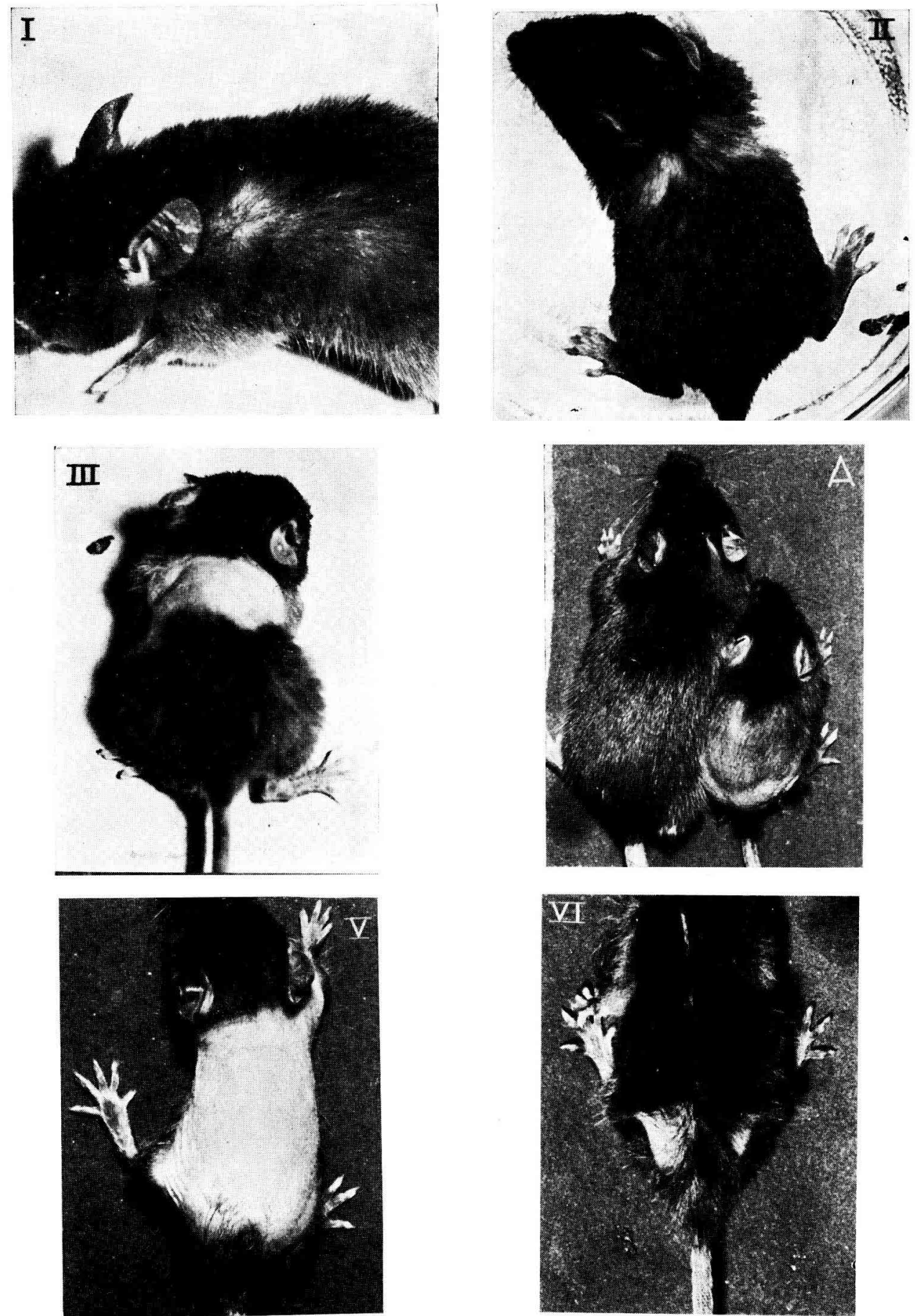

FIg. I. - Quelques types d'épilation chez la souris atteinte d'hypotrichose périodique. Type I, poils clairsemés Type II, omoplates dénudées; Type III, zone dénudée se propageant de la région scapulaire à toute la région thoracique; Type V, épilation presque complète, 'Type VI, épilation au niveau des cuisses; A, différence pondérale entre deux sujets de la même portée. 


\section{Deuxième épilation.}

Le début de cette deuxième épilation est très variable, tantôt elle s'opère quelques jours après la reprise d'aspect normal du sujet atteint ( $38^{\mathrm{e}}$ jour environ), tantôt nettement plus tard et parfois seulement vers le $60^{\mathrm{e}}$ jour. Elle se produit beaucoup plus rarement que la première et offre un aspect beaucoup moins grave et moins régulier dans son évolution ; elle est parfois suivie d'une troisième et d'une quatrième épilation encore plus légères et plus rares - poils clairsemés sur tout ou partie du corps, aires dénudées.

Cette anomalie présente donc des degrés très différents, non seulement en ce qui concerne son aspect au moment de la première épilation, mais encore dans le comportement ultérieur des sujets atteints - ainsi seule une faible proportion de ceux-ci présente une $2^{\mathrm{e}}$ épilation, encore plus rares sont ceux en ayant subi une $3^{\mathrm{e}}$ ou une $4^{\mathrm{e}}$. A noter que ces dernières ne s'observent que chez les animaux ayant subi une première épilation. Il semble donc que plus l'animal grandit et se fortifie, plus il est vigoureux et plus il est capable de lutter contre les manifestations de 1'anomalie.

\section{Étude génétique}

Le résultat des 9 types de croisements effectués (voir tableau I), montre que les sujets atteints d'hypotrichose se comportent comme des homozygotes récessifs ; l'affection est récessive à récessivité incomplète et à pénétrance extrêmement faible.

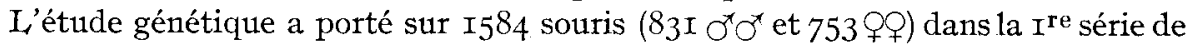
croisements et $I 667$ souris $\left(856 \sigma^{x} \sigma^{x}\right.$ et 8 I I $\left.q 9\right)$ dans la $2^{\mathrm{e}}$. Mais tous ces animaux n'ont pas été pris en considération, certains étant morts avant d'atteindre l'âge auquel apparaissent les premiers symptômes de l'anomalie - dans la Ire sétie la mortalité a été de 5,9 p. Ioo (95 souriceaux dont $44 \sigma^{x} \sigma^{x}$ et 5 I 90 ) et de Io,2 p. Ioo dans la $2^{\mathrm{e}}$ série (I7I souriceaux dont $750^{x} 0^{x}$ et 96 90 ).

Nous avons donc retenu I479 souris dans la Ire série (expérience de I àV inclus) dont 1474 normaux ( $774 \sigma^{x} \sigma^{x}$ et 700 QQ) et I 5 anormaux ( $13 \sigma^{x} \sigma^{x}$ et 2 OQ) fournis par I58 couples qui ont donné 222 portées. Dans la $2^{\mathrm{e}}$ série (expérience VI à IX inclus) comprenant I 496 souris dont II64 normat1x ( 6 I5 $\sigma^{x} \sigma^{x}$ et 549 OQ) et $33^{2}$ anormaux ( $166 \sigma^{\top} \sigma^{\top}$ et $\mathrm{I} 66$ oᄋ) fournis par I 86 couples qui ont donné 246 portées.

L'analyse génétique des 6 premiers types de croisements permet de voir :

- que la descendance de la première génération $\left(\mathrm{F}_{1}\right)$, issue du croisement $\mathrm{A} n \times \mathrm{N}$ est exclusivement normale,

- que la descendance en $\mathrm{Fb}$ (croisement de retour — père $\times$ fille ou mère $\times$ fils), est composée de 2 classes dont 2 p. roo d'anormaux,

- que dans le deuxième croisement de retour $\mathrm{F} b_{2}$ (grand-père $\times$ petite-fille), la descendance est également composée de 2 classes dont $2 \mathrm{p}$. cent d,anormaux,

- que la descendance en $\mathrm{F}_{2}$ (issue de l'expérience $\mathrm{I}$ - croisement $\mathrm{A} n \times \mathrm{N}$ ), est également composée de 2 classes dont 0,6 p. roo d'anormaux,

- que la descendance issue de parents provenant de l'expérience $\mathrm{II}(\mathrm{F} b \mathrm{~N} \times \mathrm{F} b \mathrm{~N}$ est exclusivement normale,

- enfin, que la descendance issue de 2 anormaux est composée de 2 classes dont 30,I p. Ioo d'anormaux.

Ce qui confirme bien que nous sommes en présence d'une mutation récessive, à récessivité incomplète et à faible pénétrance. 
L'analyse génétique de la descendance d'apparence normale (Nov), issue de deux anormaux se comporte génotypiquement comme des homozygotes récessifs, bien que le phénotype ne soit pas exprimé.

Ainsi, la descendance normale issue du croisement de deux anormaux (expérience VI), quel que soit de croisement auquel on la soumet (croisement de retour expérience VII, ou croisement entre deux Nov de $\mathrm{F}_{1}$ - expérience VIII, ou encore croisement entre deux Nov $\mathrm{F}_{2}$ - expérience IX), se compose de 2 classes où la proportion d'anormaux est bien plus élevée que dans les croisements de la $\mathrm{I}^{\mathrm{e}}$ série - ce qui ressort de la comparaison des types de croisements correspondants :

TABLEAU I

Constitution génotypique des souris atteintes d'hypotrichose périodique

\begin{tabular}{|c|c|c|c|c|c|c|c|c|c|c|c|c|}
\hline \multirow{4}{*}{ 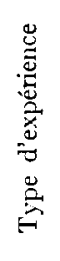 } & \multirow[t]{2}{*}{ Croisements } & \multirow{4}{*}{ 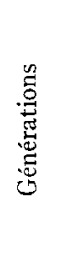 } & \multirow{4}{*}{ 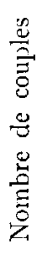 } & \multirow{4}{*}{ 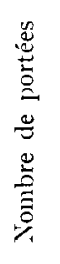 } & \multicolumn{6}{|c|}{ Descendants } & \multirow{4}{*}{$\%$} & \multirow{4}{*}{ 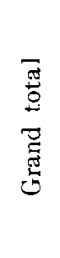 } \\
\hline & & & & & & & près 1 & jour & & & & \\
\hline & \multirow{2}{*}{$\begin{array}{l}\text { Parents } \\
\delta \hat{0} \times 9 \phi 9\end{array}$} & & & & \multicolumn{3}{|c|}{ Normaux } & \multicolumn{3}{|c|}{ Anormaux } & & \\
\hline & & & & & $0^{2} 0^{*}$ & qo & Total & $\partial^{x} \sigma^{*}$ & $q q$ & Total & & \\
\hline I & $\begin{array}{l}\text { a) } \mathrm{A} n \times \mathrm{X} \\
\mathrm{N} \times \mathrm{A} n \ldots \ldots\end{array}$ & $\mathrm{F}_{1}$ & 23 & 30 & 92 & 88 & 180 & - & - & - & 0 & 180 \\
\hline II & $\begin{array}{l}\mathrm{A} n \times \mathrm{F}_{1} \text { du } \mathrm{I} a \\
\mathrm{~F}_{1} \mathrm{~N} \text { du } \mathrm{I} a \times \mathrm{A} n \ldots . .\end{array}$ & $\mathbf{F} b$ & 36 & ‘9 & 159 & 179 & 338 & 7 & - & 7 & 2 & 345 \\
\hline III & $\mathrm{A} n \times \mathrm{F} b \mathrm{~N}$ du $\mathrm{II} a \ldots \ldots$ & $\mathrm{F} b_{2}$ & 25 & 32 & 138 & $10^{\prime}$ & $\because 42$ & 4 & 1 & 5 & 2 & 247 \\
\hline IV & $\begin{array}{l}\mathrm{F}_{1} \mathrm{~N} \text { du } \mathrm{I} a \times \mathrm{F}_{1} \mathrm{~N} \text { du } \mathrm{I} a \\
\mathrm{~F}_{1} \mathrm{~N} \text { du } \mathrm{I} b \times \mathrm{F}_{1} \mathrm{~N} \text { du } \mathrm{I} b \ldots\end{array}$ & $\mathrm{F}_{2}$ & 41 & 60 & 246 & 221 & 470 & 2 & 1 & 3 & 0,6 & 473 \\
\hline $\mathrm{V}$ & $\mathbf{F} b \mathrm{~N}$ du II $\times \mathbf{F} b \mathrm{~N}$ du II $\ldots$. & $\mathrm{F}_{2}$ bis & 33 & 51 & 139 & 105 & 241 & - & - & - & 0 & 244 \\
\hline $\begin{array}{l}\text { I ̀̀ } \\
\mathrm{V}\end{array}$ & Total & & 158 & 222 & 774 & 700 & 1474 & 13 & 2 & 15 & 1 & 1489 \\
\hline
\end{tabular}

Analyse génétique de la descendance normale (Nov)

\begin{tabular}{|c|c|c|c|c|c|c|c|c|c|c|c|c|}
\hline VI & $\mathrm{A} n \times \mathrm{A} n \ldots \ldots \ldots \ldots \ldots$ & - & 53 & 81 & 163 & 169 & 332 & 75 & 68 & 143 & 30,1 & 475 \\
\hline VII & $\begin{array}{l}\text { An } \times \mathrm{F}_{1} \text { Nov du VI } \\
\mathrm{F}_{\mathbf{1}} \mathrm{N} o v \text { du VI } \times \text { An... }\end{array}$ & $\mathbf{F}_{b}$ & 46 & 55 & 128 & 115 & 243 & 34 & 43 & 77 & 24 & 320 \\
\hline III & $\begin{array}{l}\mathrm{F}_{1} \text { Now du } \text { VI } \times \\
\mathrm{F}_{1} \text { Nov du VI } \ldots \ldots\end{array}$ & $\mathrm{F}_{2}$ & 43 & 60 & $15 \%$ & 138 & 292 & 41 & 39 & 80 & 21,5 & 372 \\
\hline II & $\begin{array}{l}F_{1} \text { Nov du VIII } \times \\
F_{1} \text { Nov du VIII } \ldots\end{array}$ & $F_{3}$ & $4^{\prime} 4$ & 50 & 170 & 127 & 297 & 16 & 16 & 32 & 9,7 & 329 \\
\hline & Total. & & 186 & 246 & 615 & 549 & $116^{\prime}$ & 166 & 166 & 332 & 22,3 & 1496 \\
\hline & Gral & & 344 & 468 & 1389 & 1249 & 2638 & 179 & 168 & 347 & 11,6 & 2985 \\
\hline
\end{tabular}


Fb (exp. VII) 24 p. Ioo d'anormaux contre Fb (exp. II) 2 p. IoO ou encore,

$\mathrm{F}_{2}$ (exp. VIII) 2I,5 p. I0o d'anormaux contre $\mathrm{F}_{2}$ (exp. IV) o,6 p. Ioo.

La fréquence nettement plus élevée d'anormaux confirme que nous sommes bien en présence de sujets "normal overlaps »; de plus la légère baisse de pourcentage en $\mathrm{F}_{1}$ et $\mathrm{F}_{2}$ est due à l'accumulation de gènes modificateurs inhibiteurs dont le rôle est encore plus marqué en $\mathrm{F}_{3}$.

\section{Distribution des sexes.}

Cette affection ne semble pas liée au sexe. Sur 347 sujets atteints, nous trouvons I79 ơ $0^{x}$ et I68 90 .

\section{Diversité phénotypique et fréquence de manifestation}

Nous constatons (tableau 2), que la fréquence de manifestation des différentes formes de l'anomalie chez les $\sigma^{x} \sigma^{x}$ et chez les $q \uparrow$ suit la formule suivante :

$$
\begin{gathered}
\sigma^{x}: \mathrm{V}>\mathrm{II} \geqslant \mathrm{I}=\mathrm{IV}>\mathrm{III}>\mathrm{VI} \\
+\mathrm{O}: \mathrm{V}>\mathrm{I}>\mathrm{IV} \geqslant \mathrm{II}>\mathrm{III}>\mathrm{VI} \\
\text { TABLEAU } 2 \\
\text { Diversité phénotypique el fréquence }
\end{gathered}
$$

\begin{tabular}{|c|c|c|c|c|}
\hline Types & Caractéristiques phénotypiques & \multicolumn{2}{|c|}{$\begin{array}{l}\text { Nombre de sujets } \\
\text { et pourcentage }\end{array}$} & $\begin{array}{l}\text { Total des } \\
\text { sujets }\end{array}$ \\
\hline \multirow{2}{*}{1} & \multirow{2}{*}{$\begin{array}{l}\text { Hipilation très légère, pas de zone dénudée, poils clair- } \\
\text { semés sur tout ou partie du corps }\end{array}$} & $\delta$ & $22(14,5)$ & \multirow{2}{*}{$\begin{array}{l}46 \\
(14,9)\end{array}$} \\
\hline & & 9 & $24 \quad(15,4)$ & \\
\hline \multirow{2}{*}{ II } & $\begin{array}{l}\text { Epilation légìre se cantonnant à la région scapulaire et } \\
\text { laissant une petite zone dénudée sur l'omoplate }\end{array}$ & $\partial$ & $23 \quad(15,2)$ & \multirow{2}{*}{$\begin{array}{l}38 \\
(12,3)\end{array}$} \\
\hline & gauche ou droite ou sur les deux & q & $15 \quad(9,6)$ & \\
\hline \multirow{2}{*}{ III } & \multirow{2}{*}{$\begin{array}{l}\text { Epilation plus marquée, se propageant de la région } \\
\text { scapulaire ì toute la région thoracique }\end{array}$} & o & $1 ; \quad \quad(9,2)$ & \multirow{2}{*}{$\begin{array}{l}26 \\
(8,4)\end{array}$} \\
\hline & & q & $12 \quad(7,7)$ & \\
\hline \multirow[t]{2}{*}{ IV } & $\begin{array}{l}\text { Épilation plus nette gagnant progressivement sur les } \\
\text { précédentes et atteignant la région lombaire à }\end{array}$ & $\sigma$ & $22 \quad(1 ' k, 5)$ & \multirow{2}{*}{$\begin{array}{l}38 \\
(12,3)\end{array}$} \\
\hline & laquelle elle s'arrête — animal dénudé aux $3 /{ }^{\prime} t$ & q & $16 \quad(10,3)$ & \\
\hline \multirow[t]{2}{*}{$\mathrm{V}$} & \multirow{2}{*}{$\begin{array}{l}\text { Epilation presque complète, seules restent couvertes } \\
\text { de poils la tête et la base de la queue }\end{array}$} & $\hat{o}$ & $67 \quad(4,4,3)$ & \multirow{2}{*}{$\begin{array}{l}152 \\
(49,3)\end{array}$} \\
\hline & & q & $85 \quad\left(5^{\prime}, 8\right)$ & \\
\hline \multirow{4}{*}{ VI } & $\begin{array}{l}\text { Épilation exclusivement cantonnée au niveau des } \\
\text { cuisses, le reste du corps est normalement couvert }\end{array}$ & 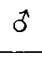 & $3 \quad(1,9)$ & \multirow{2}{*}{$\begin{array}{l}6 \\
(1,9)\end{array}$} \\
\hline & de poils. & q & $3 \quad(1,9)$ & \\
\hline & \multirow{2}{*}{ Total } & $\hat{\sigma}$ & $151 \quad(49,3)$ & \multirow{2}{*}{306} \\
\hline & & q & $155 \quad(50,6)$ & \\
\hline
\end{tabular}


Nous voyons donc que la fréquence la plus élevée est celle de la forme la plus grave (Type $\mathrm{V}$-épilation presque complète), le taux de $\bigcirc \bigcirc$ atteintes est plus grand que celui des $\sigma^{x} \sigma^{x}(54,8$ contre 44,3 p. I00). La fréquence la plus faible est celle du Type VI-épilation exclusivement cantonnée au niveau des cuisses, là le taux de $\sigma^{\top} \sigma^{\top}$ atteints est sensiblement égal à celui des $\not \varnothing$; très faible $(\mathrm{r}, 9 \mathrm{p}$. 100). L,es formes intermédiaires se manifestent à peu près de la même façon chez les ơ ơ et chez les $q$.

\section{Anomalies concomitantes.}

Dans de rares cas nous avons vu apparaître des anomalies associées à l'hypotrichose ; il s'agit de : I) panachure ombilicale, qui se prête à la sélection, 2) nanisme, 3) anomalies podales (raccourcissement de l'humérus ou du fémur), cette dernière ne semble pas héréditaire.

Nous avons constaté que les facteurs " parturition " et "saison " ont eux aussi une influence certaine sur la fréquence d'apparition de cette anomalie.

\section{CONCLUSIONS}

L'hypotrichose périodique est une mutation récessive à récessivité incomplète et à faible pénétrance, qui se manifeste entre le $\mathrm{I}^{\mathrm{e}}$ et le $24^{\mathrm{e}}$ jour après la naissance, s'accompagne d'un arrêt de croissance et se répète parfois à l'occasion des autres mues, mais de façon irrégulière. I'épilation débute dans la région scapulaire et s'y cantonne ou progresse par vagues successives vers la base de la queue.

Le phénotype est très variable et va des formes les plus légères - poils clairsemés sur tout ou partie du corps - aux formes les plus graves, épilation s'étendant jusqu'à la région sacrée, seules restent couvertes la tête et la base de la queue, ou encore une forme tout à fait aberrante d'épilation cantonnée au niveau des cuisses.

Notre mutation peut être rapprochée de diverses anomalies du poils qui ont été observées tant chez la souris que dans les autres espèces animales, domestiques ou sauvages, ainsi que chez l'homme. Elles sont d'ordre mutationnel, le plus souvent à caractère récessif, plus rarement à caractère dominant. Ces anomalies portant sur la chute plus ou moins complète des poils, temporaire comme dans 1' " hypotrichosis juvenilis " de LOEFFLER, ou définitive dans les formes telles que " alopécia » de Dickie, ou de SUMner, ou " rhino " de Howard et Fraser, ou encore "naked " et " hairless ", sont souvent mentionnées dans la littérature.

L'hypotrichose périodique que nous avons décrite est comparable à l'« hypotrichosis juvenilis " de LOEFFLER tant pour ce qui est de l'aspect phénotypique de la première mue anormale que pour l'époque à laquelle celle-ci intervient; mass la récessivité de notre mutation est incomplète et de plus elle se répète parfois à l'occasion des autres mues; chez LOEFFLER, au contraire, la récessivité est complète, pas de " normal overlaps".

L'analyse génétique montre que les sujets atteints d'hypotrichose se comportent comme des homozygotes récessifs. L'étude de la descendance normale, issue de deux anormaux (pénétrance 30,I p. Ioo) montre que ces sujets "normal overlaps " se comportent comme des anormaux; dans le croisement $\mathrm{A} n \times$ Nov la pénétrance 
est légèrement plus faible ( $24 \mathrm{p}$. Ioo), elle n'est que de $2 \mathrm{I}, 5 \mathrm{p}$. Ioo dans le croisement Nov $\times$ Nov, issus de deux anormaux et en $\mathrm{F}_{2} \mathrm{Nov} \times \mathrm{F}_{2} \mathrm{Nov}$ de $9,7 \mathrm{p}$. Ioo seulement. Par ailleurs, on peut constater dès maintenant que d'autres facteurs ont une influence certaine sur l'apparition de l'anomalie, ils méritent une étude plus détaillée, ce sont le rang chronologique de la parturition (influence de la $I^{\mathrm{er}}, 2^{\mathrm{e}}, 3^{\mathrm{e}}$ parturition), et la saison à laquelle celle-ci intervient.

Reçu pour publication en avril 1963.

\section{SUMMARY}

\section{PERIODICAL HYPOTRICIOSIS IN MICE}

The periodical hypotrichosis in mice is a recessive mutation with incomplete penetrance. In this work we used 2985 mice $\mathrm{C}_{57} \mathrm{BL} / \mathrm{J} a x 6$ and nine types of crossing have been carried out.

The genetical analysis of the normal progeny of abnormal breeding pairs (penetrance $30,3 \mathrm{p}$. 100, show that they behave genetically as abnormal subjects. In the backcross $a n \times$ Nov (abnormal $\times$ normal overlaps), the penetrance is lower $(24 \mathrm{p}, 100)$, and if we cross Nov $\times$ Nov offspring of two abnormal parents it is but $2 \mathrm{I} .5$ per cent, while in the $\mathrm{F}_{2} \mathrm{Nov} \times \mathrm{F}_{2} \mathrm{Nov}$ it is no more than 9.7 per cent ; here are interfering inhibitory modifying genes.

The phenotypical manifestation take place between the I6th and 2 Ist day after birth and sometimes a second, third or even fourth loss of hair can be observed at times of other sheddings, but they are obviously less frequents.

The loss of hairs (shedding of the first coat), starts at the scapular region and spreads rapidly, like advancing waves, towards the tail. During this time the abnormal subjects lag behind their normal sibs in growth but catch up later in life.

The phenotype is variable, there are 6 classes of first abnormal shedding :

- scarce loss of hair, hair scarce on the whole or part of the body (Type I),

- loss of hair in the scapular region (Type II),

- loss of hair on the whole thoracic region (Type III), the tail (Type V),

- loss of hair on the thighs and later than normally (Type VI).

They recover hair and weight and at the 4oth day aprox. after birth there remain no noticable difference between normal and abnormal subjects of the same litter.

Two factors are also interfering in the frequency of litters with abnormal subjects : the rank of parturition and season when it takes place.

\section{RÉFÉRENCES BIBLIOGRAPHIQUES}

Auerbach Ch., Falconner D. S., I949. A new mutant in the progeny of mice treated with nitrogen mustard. Nature, 163, 678-679.

Bonnet R., т 892 . Über Hypotrichosis congenita universalis. Anat. Hefte, 1, 233-270.

Brain R. T., i938. Alopecia congenita : minor ectodermal defect. Proc. Roy. Soc. Med., 32, 87-88.

Brooke H. C., 1926. Hairless mice. J. Hered., 17, 173-1 74.

Carter T. C., Philips R. S., 1949. Ichthyotic mice. Heredity, 3, 379 (Abstr.).

Carter T. C., Philips R. S., 1954. Ragged, a semidominant coat texture mutant in the house mouse. $J$. Hered., 45, $5 \mathrm{I}$.

Castle W. E., Dempster E. R., Shurrager H. C., ig55. Three new mutant of the rat. J. Hered. 46, 9-I 4 .

Cole L. J., I9I9. A defect of hair and teeth in cattle - probably hereditary. J. Hered., 10, 303-306.

Craft W. A., Blizzard W. L., I934. The inheritance of semi-hairlessness in cattle. J. Hered., 25, 384-39o.

Crew F. A. E., 1927. The hairless mouse. Rep. Brit. Ass., Leeds : 335 . 
Crew F. A. E., Mirskaia L., I932. The character " hairless " in the mouse. J. Genet, 25, I 7-24.

Cupp M. B., 1958. "Sparse-fur" - cité d"après Mouse News Letter 19, 37.

David L. T., I931. Hairless mammals. Comparative histologic studies preliminary report. Arch. Dermat. a. Syph., 24, I96-203.

DAvid L. T., 1932. The external expression and comparative dermal histology of hereditary hairlessness in mammals. Z. Zellforsh., 14, 6r6-7 19.

David L. T., I932. Histology of the skin of the Mexican Hairless Swine (Sus scrofa). Am. J. Anat., 50, $283^{-292 .}$

Dickie M. M., 1955. Alopecia, a dominant mutation in the House Mouse. J. Hered., 46 (n' 1 ), 30-34.

Dickie M. M., Kelton E. D., Fielder J. H., Ingalls A. M., Snell C. D., I949. New mutations and linkage studies in the house mouse (Mus musculus). Anat. Rec., 105, 540.

Drieux H. M., Priouzeau C., Thiery, Priouzeau M. L., Hypotrichosis congénitale avec anodontie, acérie et macroglossie chez le veau. Rec. Med. Vét. 126, 385-399.

EBbenhorst TengBergen W. J. P. R., I939. Lethality and abnormal condition of the pelage of the Latvian mouse (dominant Naked). Genetica, 21, 369-385.

EISELE F., I936. Angeborene Haarlosigkeit bei Kälbern des schwarzbunden Niederungszucht als Auswirkungeines Letalfaktors. Züchtungskunde, 11, 432-437.

Eldince F. E., Atkeson F. W., 1953. Streaked hairlessness in IIolstein Friesian cattle. J. Hered., 44, $265-27 \mathrm{I}$.

Fiscirer E., igıo. Ein Fall von erblicher Ilaararmut und die Art ihrer Vererbung. Arch. f. Rass. Ges. Biol., 7, 50-56.

FRASER T. C., 1946. The expression and interaction of hereditary factors producing hypotrichosis in the mouse : histology and experimental results. Canad. J. Res. D., 24, 10-25.

Furlotri A., igio. Sopra un caso di mancata formazione del pelo in una Talpa europea. L. Zool. Ann., 36, $125-\mathrm{I} 32$.

Garber E. D., I952. "Bald a second allele of hairless in the house mouse. J. Hered. 43, 45-46.

Gates R. R., 1948. Human Genetics. The Macmillan Company, New York.

Gillepsie J. B., I937. Congenital and familial alopecia totalis. Am. J. Dis. Child., 53, 132-г 36.

Gordon, 1850, cité d'après DAvid L. T.

Gowen J. W., 1962. Genetic patterns in senescence and infection. J. Amer. Ger. Soc., 10, 107-I 24.

Green E. L., 1954. The genetics of a new hair deficiency, furless. J. Hered., 45 (n 3), i I 5-I I 8.

Grunererg II., 1952. The genetics of the mouse. Ed. Martinus Nijhoff.

Hollander W. F., Gowen J. W., 1959. A Single-gene Antagonism between mother and Fetus in the Mouse. Proc. Soc. Exp. Biol. Med., 101, 425-428.

HowarD A., I940. "Rhino ", an allele of hairless in the house mouse. J. Hered., 31, 467-470.

Hunsicker P. R., ig6o, cité d'après Mouse News Letter, 23, 58-59.

Hutt F. B., Sanders L. Z.., I953. Viable genetic Hypotrichosis in Guernesey cattle. J. Hered., 44 (3), 97-103.

Kidweld J. F., Guilbert H. R., i950. A recurrence of semi-hairless gene in cattle. J. Hered., 41, igo192.

Kislovsky D. A., I928. Naked - a recessive mutation in the rabbit. J. Hered., 19, 438-439.

KoboziefF N., Pomriaskinskx-KoboziefF N. A., i943. Précis de génétique appliquée de la médecine vétérinaire, Vigot Frères - Paris.

Kobozieff N., Pomriaskinsky-kobozieff N. A., Drieux H. I958. Alopécie héréditaire, associée à d'autres affections chez la souris - note préliminaire.Rec. Med. Vét., 134, 375-384.

Krallinger H. F., I955. Angewandte Vererbungslehre für Tierzüchter. Eugen. Ulmer, Stuttgart.

Lebedinsky N. G., DaUwart A., 1927. Atrichosis und ihre Vererbung bei der albinotischen Hausmaus. Biol. Zbl., 47, 748-752.

Letard E., I930. L’hérédité mendélienne du caractère " peau nue " chez les chiens. C. R. Soc. Biol., 103, I $135-1136$.

Letard E., I938. Hairless Siamese cats. J. Hered., 29, I73.

Liutikov K. M., I 938. Hairlessness in the animal Kingdom. Uspekhi Sovremenoi Biol., 9, 269-286.

LOEFFler L., 1934. uber eine neue Form von Hypotrichosis (IIypotrichosis juvenilis), bei der weissen Hausmaus. Ziav., 67, 209-211.

Lyon M. F., 1956. Hereditary hair loss in the tufted mutant of the house mouse. J. Hered., 47, Ior-Io3.

Mann S. J., Stralte W. E., I96r. New observations on Hair loss in the hairless mouse. Anat. Rec., 140, 97-102,

Marsinal C. C., I887. Hairless mice in Humboldt country. West. Amer. Scientist (San Diego, Cal.), 3, 72-73.

Melien I. M., 1939. The origin of the Mexican hairless cat. J. Hered., 30, 435 .

Monr O. L., Wriedt C., I928. Hairless, a new recessive lethal in cattle. J. Genetics, 19, 315-336.

NAY T., I960. Growth of the mouse coat VII. Hair cycles and sebaceous glands in homozygous and heterozygous naked mice. Aust. J. Biol. Sci., 13, 351-355. 
Packchanian A., Sinclair J. G., I 959. The rearing of homozygous hairless deer mice, Peromyscus maniculatus gambeli, with a note of superfetation or delayed fertilization. Tex. Rep. Biol. Med., 17, 229-236. Pizarro O., 1957. Cité d'après Mouse News Letter, 17, 96.

Redlichs A., I937 a. Über die Hauthistologie der Haarlosen lettländischen Maus. Bull. Soc. Biol. Lett., 7., IO3-II3.

ReDlichs A., I 937 b, Dermal histology of the Latvian " nacked mouse 》. Biol. generalis, 13, 504-528.

Regan W. M., Mead S. W., Gregory P. W., I935. An inherited skin defect in cattle. J. Hered., 26, 357362 .

Roberts E., I924. Inheritance of Hypotrichosis in rats. Anat. Rec., 29, I4I.

Roberts E., Carrol N. E., I93r. The inheritance of "Hairlessness " in swine. Hypotrichosis II. J. Hered. 22, $125-132$.

Rosenberg G., I939. Angeborene Haarlosigkeit (Hypotrichosis adnata) bei einem Kalb. Deut. Tierärztl. Woch., 47, 21 2-213.

Scheurer O., Kohn F. G., rgir. Vergleichende Befunde bei Hypotrichosis des Menschen und des Hundes. Arch. Dermat. u. Syph., 109, 79-101.

Searle A. G., Spearman, 1957. "Matted " a new hair mutant in the house mouse. Genetics and Morphology. J. Embr. Exp. Morph., 5, 93-102.

Shibata S., Ishinara M., r949. Studies on the hereditary defects in Japanese native cattle. Jap. J.Zootech. $S c i, 19,63^{-66 .}$

SleE J., 1957. The morphology and development of Ragged - a mutant affecting the skin and hair of the house mouse. J. Genetics, 55, 570-584.

SNELL G. D., I93I. Inheritance in the house mouse, the linkage relations short-ear, hairless and nacked. Genetics, 16, 42-74.

Steinberg A. G., Fraser T. C., I943. The interaction of rhino with naked hairless. Genetics, 28, $9 \mathrm{l}-92$

Steinberg A. G., Fraser T. C.,I946. The expression and interaction of hereditary factors affecting hair growth in mice ; external observations. Canad. J. Res., D. 24, I-9.

Sumner F. B., 1924. Hairless mice. J. Hered., 15, 475-48r.

Surrarre T. C., I943. Bulldogs and hairless calbes. J. Hered., 34, 175-1 78.

Tegetmeier, I876, cité d'après David L. T.

Thorner J. E., I940. Alopecia totalis. Endocrinology., 26, 433-436.

Waelsci L., igro. Über Hypotrichosis (Alopecia congenita). Arch. Dermat. u. Syph., 103, 63-92.

Wipprecit C., Horlacher W. R., 1935. A lethal gene in Jersey cattle. J. Hered. 26, 363-368. 